\title{
TOPOLOGICAL REPRESENTATION OF ALGEBRAS
}

\author{
BY \\ RICHARD F. ARENS AND IRVING KAPLANSKY
}

1. Introduction. Stone $[23$, Theorem 1$]\left(^{(}\right)$has shown that a Boolean ring with unit is the set of all open and closed sets in a compact (=bicompact) zero-dimensional space. In slightly different terminology: a Boolean ring with unit is the set of all continuous functions from a compact zero-dimensional space to the field $G F(2)$ of two elements.

Gelfand [6] and others have obtained theorems which assert that a Banach algebra, under suitable conditions, is the set of all real-valued or complexvalued continuous functions on a compact Hausdorff space.

In both of these contexts we have the following situation: an algebra $A$ is given over a field $K$, and hypotheses are imposed which make certain that for every maximal ideal $M, A-M$ is isomorphic to $K$. In the first case $K$ is $G F(2)$, in the second the reals or complexes.

The present paper is devoted to exploration of the consequences of relaxing the demand that all residue fields be $K$. The results can be well illustrated by discussing the case of a ring $A$ (say with unit) in which every element satisfies the equation $a^{4}=a$. Here the residue fields may be $G F(2)$ or $G F(4)$; in any event they are all subfields of $G F(4)$. We can at once represent $A$ as a set of functions from the class $X$ of maximal ideals to $G F(4)$. But if we wish to obtain a theorem that asserts that we get all functions of a prescribed kind, we must introduce topological qualifications. We topologize $X$ after Stone, getting a compact zero-dimensional space (structure space of $A$ ). Now we face the fundamental difficulty. The field $G F(4)$ admits an automorphism, and so there is an essential ambiguity in setting up the mapping $A-M \rightarrow G F(4)$ at each point (maximal ideal) where $A-M$ is $G F(4)$. If we simply make an arbitrary choice at each point, we will jeopardize the hoped for continuity of the functions. It is to be observed that this difficulty does not arise in setting up a purely algebraic representation, say as a subdirect sum.

The question is thus posed: is it possible to make a simultaneous choice of mappings at all the points in such a way as to render the functions continuous? The answer is in general negative: a counter-example is given in $\S 8$. This ring cannot be represented by a ring of continuous functions on its structure space to $G F(4)$ at all.

However with further restrictions on $A$ the answer is affirmative: for example, if $A$ is countable ( $X$ then has the second axiom of countability). Our structure theorem in this case reads: $A$ is the set of all continuous func-

Presented to the Society, April 25, 1947; received by the editors March 12, 1947.

(1) Number in brackets refer to the bibliography at the end of the paper. 
tions from $X$ to $G F(4)$, restricted on a closed set to values in $G F(2)$.

To obtain a successful representation in the general case, we abandon the structure space itself as a domain for functional representation. We form the Kronecker product $B=A \times G F(4)$, that is, the set of all $b+c j$, with $b, c \in A$ and $j^{2}+j+1=0 . B$ is an algebra over $G F(4)$, and each of its elements again satisfies the equation $a^{4}=a$. There is no difficulty in representing $B$ as the set of all continuous functions from its structure space $Y$ to $G F(4)$; the previous obstacle has disappeared since the presence of $G F(4)$ as a field of scalars induces a natural mapping of $B-M$ into $G F(4)$. The automorphism $b+c j$ $\rightarrow b^{2}+c^{2} j$ induces a homeomorphism $\theta$ of $Y$, and it can be seen that $A$ is precisely the set of functions $f$ for which $f(y \theta)=[f(y)]^{2}$ for $y \in Y$. Thus by using the larger space $Y$, our representation does yield continuous functions, but at the expense of being restricted to functions bearing a special relation to a homeomorphism of $Y$.

Similar ideas are applied in $\S 9$ to the case of a Banach algebra $A$ over the reals. Here $A-M$ may be the field of reals or complexes. In the latter case we again face an ambiguity, the automorphism in question being of course complex conjugation. Passage to the Kronecker product yields the following structure theorem: $A$ is the set of all continuous complex-valued functions on a compact Hausdorff space $X$, subject to the restriction that $f(x \theta)$ and $f(x)$ are conjugates, where $\theta$ is a homeomorphism of $X$ whose square is the identity. Thus we obtain representation theorems for Banach algebras which provide a sort of continuous transition from those of Gelfand and Neumark [7], and those with exclusively real residue fields [ 1 or $6, \mathrm{pp} .15-16]$. If $\theta$ is the identity, the functions are real; if $\theta$ interchanges two disjoint open and closed sets $X_{1}, X_{2}$, we have all complex functions, say on $X_{1}$; in general we have some intermediate case.

Introductory sections ( $\$ 2-4)$ are devoted to preliminary results. Many of these can be obtained for fairly general classes of rings which we introduce: biregular and strongly regular. The link with algebraic algebras is affected in Theorem 3.3, and the succeeding material leads up to our main theorem (6.1), which extends the kind of result described above to any commutative semisimple algebraic algebra. In $\$ \S 7-8$ we obtain the simplified representations made possible by countability assumptions.

2. Biregular rings. We define a biregular ring to be a ring in which every principal two-sided ideal is generated by an idempotent element in the center, that is, for any $a$ we have $(a)=(e)$ where $e$ is an idempotent in the center, and (a) denotes the smallest two-sided ideal containing $a$. A Boolean ring is biregular since all its elements are idempotents in the center. As we shall see, many of Stone's results on Boolean rings can be extended to biregular rings.

Biregular rings may also be regarded as the two-sided analogue of von Neumann's regular rings [20], where every principal right ideal is generated by an idempotent (not necessarily in the center). We shall give examples to 
show that the two concepts are independent.

(a) A regular ring which is not biregular: the ring of all linear transformations in an infinite-dimensional linear space. (For a proof of regularity, cf. [14].) If $a$ is a nonzero linear transformation with finite-dimensional range, then $(a)$ is the ideal of all linear transformations with finite-dimensional range, while on the other hand the only idempotents in the center are 0 and 1.

(b) A biregular ring which is not regular: the ring of differential polynomials discussed on p. 237 of [11]. It is a simple ring with unit and hence biregular (Theorem 2.1 below); however since it has no divisors of zero, it cannot be regular.

For commutative rings, or for rings with the descending chain condition on right ideals, the concepts of regularity and biregularity coincide.

THEOREM 2.1. A biregular ring is semi-simple $\left({ }^{2}\right)$. It is primitive if and only if it is a simple ring with unit.

Proof. The first statement follows from the fact $[10$, p. 305] that the only idempotent in the radical is 0 . Suppose that the biregular $\operatorname{ring} A$ is primitive, and let $e$ be an idempotent in the center. If $B$ is the ideal consisting of all $x-e x$, we have $(e) B=0$. By [10, Lemma 4$]$, either $(e)$ or $B$ is 0 , that is to say, $e=0$ or 1 . Hence $A$ is simple with unit. The converse follows from the fact that in a simple ring with unit, 0 and (1) are the only two-sided ideals.

COROLLARY 1. In a biregular ring the concepts of primitive and maximal ideal coincide.

In any semi-simple ring the intersection of the primitive ideals is zero [10, Theorem 25]. Hence we have the following result.

COROLlary 2. A biregular ring is a subdirect sum of simple rings with unit.

We remark that the converse of Corollary 2 fails, as is already shown by the ring of integers.

It is evident that any homomorphic image of a biregular ring is biregular. An immediate consequence is the following generalization of $[22$, Theorem 66].

COROLlaRY 3. In a biregular ring every two-sided ideal is the intersection of the maximal ideals containing it.

Following Jacobson [12] we define for any ring $A$ a topological space called the structure space: its points are the primitive ideals of $A$, and (Stone's topology) the closure of a set $\left\{P_{\alpha}\right\}$ of primitive ideals is the totality of primitive ideals containing $\cap P_{\alpha}$. The structure space is always a $T_{0}$-space; in a

(2) We mean semi-simple in the sense of Jacobson [10]. The terms "radical" and "primitive" will also be used as there defined. The alternative theories of the radical in [4] or [21] could equally well be employed in the context of biregular rings. 


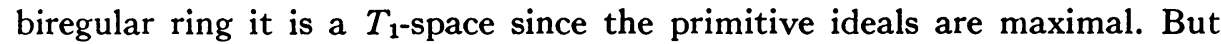
actually considerably stronger separation properties hold, as we now proceed to show.

We quote from Jacobson the following two results: (1) if $A$ has a unit its structure space is compact, (2) if $B$ is an ideal in $A$, the structure space of $A-B$ is homeomorphic to the set of primitive ideals containing $B$. In particular then, the set of primitive ideals containing $B$ is compact if $A-B$ has a unit (cf. [21, Theorem 2.9]).

Lemma 1.1. Let $e$ be an idempotent in the center of a ring $A$. Then the set $E$ of primitive ideals not containing e forms an open compact set in the structure space of $A$.

Proof. The set of primitive ideals containing $e$ is (virtually by the definition) closed; hence $E$ is open. Let $B$ be the set of all $x-e x$ and $P$ any primitive ideal. Since $(e) B=0$ we have $[10$, Lemma 4$]$ that either $(e)$ or $B$ is contained in $P$. Thus e $€ P$ is equivalent to $B \subset P$, whence $E$ is precisely the set of primitive ideals containing $B$. Since $A-B$ has a unit element, $E$ is compact.

Lemma 1.2. Let $B, C$ be ideals in a ring $A$ such that $B+C$ is not contained in any primitive ideal, and suppose further that there exists an element $e$ which is a unit element modulo $B$. Then $C$ contains a unit element modulo $B$.

Proof. We must have $B+C=A$, for otherwise (since $A-B$ has a unit) $B+C$ could be embedded in a primitive ideal. Hence $b+c=e$, with $b \in B$, $c \in C$, and $c$ is the desired unit element modulo $B$.

If $E$ is a set of primitive ideals, we call $e$ a unit modulo $E$ if it is a unit element modulo each member of $E$. We shall say that $e$ is the characteristic function of $E$ if $e$ maps into 1 modulo each primitive ideal in $E$ and into 0 modulo each primitive ideal not in $E$. Such an element can exist only if $E$ is an open compact set, and in a semi-simple ring it is necessarily unique.

Lemma 1.3. Let $A$ be a ring with structure space $S$, and $E \subset S$ an open and closed set such that $A$ contains a unit modulo $E$. Then $A$ contains a characteristic function of $E$.

Proof. Apply Lemma 1.2 with $B, C$ the intersections of the ideals in $E$, $S-E$ respectively.

LEMMA 1.4. Let $A$ be a ring whose structure space $S$ is zero-dimensional $\left({ }^{3}\right)$, and suppose that each point in $S$ has a neighborhood $U$ such that $A$ contains a unit modulo $U$. Then $A$ contains a characteristic function of any open compact set $K$ in $S$.

(3) We shall use the term "zero-dimensional space" to denote a $T_{0}$-space having a basis of open and closed sets. A zero-dimensional space is necessarily Hausdorff. It is to be noted that a locally compact Hausdorff space with no connected sets other than points is zero-dimensional [cf. 8, p. 20]. 
Proof. For each point $x$ in $K$ we may pick an open and closed neighborhood $U(x)$ such that $A$ contains a unit modulo $U(x)$. A finite number of these neighborhoods cover $K$. By decomposition and intersection with $K$ we may obtain disjoint open and closed sets $U_{1}, \cdots, U_{n}$ which precisely cover $K$. By Lemma 1.3, $A$ contains a characteristic function $e_{i}$ of each $U_{i}$. The element $e_{1}+\cdots+e_{n}$ is a characteristic function of $K$.

THEOREM 2.2. The structure space $S$ of a biregular ring $A$ is locally compact and zero-dimensional. A contains the characteristic function of any open compact set in $S$, and in particular $S$ is compact if and only if $A$ has a unit element.

Proof. Let $M, N$ be two distinct maximal ideals. We can find an element, and hence an idempotent in the center, which is contained in $N$ but not in $M$. Hence by Lemma 1.1 there exists an open compact set containing $M$ but not $N$. This proves the local compactness and zero-dimensionality. The remaining statement follows from Lemma 1.4, since we have now verified the latter's hypotheses.

Before stating the next theorem we make some preliminary remarks. Let $A$ be a biregular ring admitting a ring $R$ of left operators. Then any left ideal $I$ is automatically $R$-admissible. (This is another of the many respects in which a biregular ring resembles a ring with unit.) For let $x \in A, \lambda \in R$; if $(x)=(e)$ we have that $e x=x$ and hence $(\lambda e) x=\lambda(e x)=\lambda x$ is in $I$. If $I$ is twosided, $A-I$ admits $R$ as left operators. If further $R$ has a unit element which acts as unit operator, and $A-I$ has a unit element $e$, the mapping $\lambda \rightarrow \lambda e$ is an isomorphism between $R$ and a subring of $A-I$. In the sequel we shall speak of this as the "natural map" of $R$ into $A-I$.

THEOREM 2.3. Let $R$ be a given (discrete) simple ring with unit. The following conditions are necessary and sufficient for $A$ to be the set of all continuous functions, vanishing outside a compact set, from a locally compact zero-dimensional space $X$ to $R$ :

(1) $A$ is biregular,

(2) $A$ admits $R$ as a ring of left operators with the unit element of $R$ acting as unit operator.

(3) If $M$ is a maximal ideal in $A$, the natural map from $R$ to $A-M$ is an isomorphism of $R$ and $A-M$.

Proof. Necessity. Suppose $A$ is the ring of functions in question. It is to be noted that these functions assume only a finite number of values, each on an open compact set. Let $a$ be any element in $A$ and $e$ the characteristic function of the set where $a$ does not vanish; then $(a)=(e)$, proving the necessity of (1). The necessity of (2) is evident, and the necessity of (3) will be sustained by proving the following: every maximal ideal in $A$ consists of all functions vanishing at a point. Suppose the contrary for a maximal ideal $M$. Let $a \in A$ be any function not in $M$, and $K$ the (compact) set where it does not vanish. 
For any $x$ in $K, M$ contains a function not vanishing at $x$, hence not vanishing in an open and closed neighborhood of $x$. A finite number of these neighborhoods cover $K$; we decompose them, obtaining disjoint open and closed sets $W_{1}, \cdots, W_{n}$ which precisely cover $K . M$ contains the characteristic function of each $W_{i}$ and hence contains $a$, a contradiction.

Sufficiency. We let $X$ be the structure space of $A$. By (3) we can at once represent the elements of $A$ in a natural way as functions from $X$ to $R$, and by Lemma 1.1 each of them vanishes outside a compact set. By Theorem 2.2, $A$ contains at least all characteristic functions of open compact sets. But then it must contain all continuous functions vanishing outside a compact set, for these are linear combinations of a finite number of characteristic functions of open compact sets. It remains to be proved that all our functions are in fact continuous. Given $a \in A$, let $\lambda \in R$ be one of the values $a$ assumes; we have to prove that the set $Y$ where $a$ assumes $\lambda$ is open and closed. Let $e$ be the characteristic function of the set where $a$ does not vanish; then $Y$ is the set where $a-\lambda e$ vanishes, intersected with the set where $a$ does not vanish. By Lemma 1.1, $Y$ is open and closed.

If $A$ is a Boolean ring, the hypotheses of Theorem 2.3 are readily verified with $R=G F(2)$. We thus obtain as a special case Stone's topological representation of Boolean rings [23, Theorem 1]. In $\$ \S 5-8$ we shall obtain representation theorems under milder assumptions than those of Theorem 2.3; application can then be made to a wider class of rings, for example rings with $a^{4}=a$.

3. Strongly regular rings. We define a ring to be strongly regular if for every $a$ there exists an $x$ such that $a^{2} x=a$. It will be shown below that the definition is actually right-left symmetric, and that strong regularity implies both regularity and biregularity.

Actually many of the results can be extended to the more general class of rings discussed in the next theorem.

THEOREM 3.1. Let $A$ be a ring in which for every a there exists an $x$ such that $a^{n+1} x=a^{n}$ (with $n$ independent of $a$ ). Then the radical of $A$ is a nil-ideal with index of nilpotence at most $n$. If $A$ is primitive, it is a matrix ring of order at most $n^{2}$ over a division ring. Instances of such rings are rings with the descending chain condition on right ideals, and algebraic algebras of bounded degree $\left(^{4}\right)$.

Proof. Suppose $a$ is in the radical of $A$. Then $-a x$ is quasi-regular, say with quasi-inverse $y:-a x+y-a x y=0$. Left-multiply by $a^{n}$, and we obtain $a^{n+1}=0$ so that $a^{n}=0$.

If $A$ is primitive, it is a dense ring of linear transformations in a vector space $V$ over a division ring. Suppose $V$ has $n+1$ linearly independent elements $x_{1}, \cdots, x_{n+1} . A$ contains a linear transformation $a$ which sends $x_{1}$ into

(4) Cf. [10] for definitions. 
0 and $x_{i}$ into $x_{i-1}$ for $2 \leqq i \leqq n+1$. Then $a^{n}$ sends $x_{n+1}$ into $x_{1}$, and $a^{n+1}$ annihilates all the $x$ 's. This contradicts the existence of an equation of the form $a^{n+1} x=a^{n}$. Hence $V$ is at most $n$-dimensional, and $A$ consists of all linear transformations in $V$.

Let $A$ be an algebraic algebra whose elements have degrees bounded by $n$. Thus for any $a \in A$ we have $a^{n}+\cdots+\theta a^{k}=0(\theta \neq 0)$. We multiply by $\theta^{-1} a^{n-k}$ and obtain $a^{n}=a^{n+1} x$ with $x$ a certain polynomial in $a$ (in the extreme case $k=n, x$ may be chosen arbitrarily).

Finally, suppose $A$ is a ring with the descending chain condition on right ideals. Then $A-R$ is a direct sum of matrix rings $M_{i}$ over division rings $D_{i}$. Each element $a$ in $M_{i}$ satisfies a polynomial equation with coefficients in $D_{i}$ and hence (by the same argument as above) we have $a^{m}=a^{m+1} x$ with $m$ independent of $a$. By taking the largest of the $m$ 's, we extend the property to $A-R$. Now suppose $R^{k}=0$. We have $a^{m} \equiv a^{m+1} x \equiv a^{m+r} x^{r}(\bmod R)$ for any $r$. Take $r=m k-m+1$, and from $\left(a^{m}-a^{m+r} x^{r}\right)^{k}=0$, we deduce $a^{m k}=a^{m k+1} y$.

Let us take the case $n=1$ in Theorem 3.1 , so that $A$ is strongly regular. We deduce that $A$ is semi-simple, that any primitive ideal $M$ in $A$ is maximal, and that $A-M$ is a division ring. It follows that, for the $x$ satisfying $a^{2} x=a$, $a x$ must map into either 0 or 1 modulo a maximal ideal. Hence $a x=x a$ is idempotent and in the center. We have thus verified the statements in the following theorem.

THEOREM 3.2. A strongly regular ring is both regular and biregular, and any quotient ring modulo a maximal ideal is a division ring.

The results on strongly regular rings are applicable to certain algebraic algebras, in the light of the following theorem.

THEOREM 3.3. For an algebraic algebra, or for a ring with descending chain condition, the following are equivalent: (a) strong regularity, (b) absence of nilpotent elements $(\mathbf{5})$. For a commutative algebraic algebra, or for a commutative ring with descending chain condition, the following are equivalent: (a) regularity, (b) absence of nilpotent elements, (c) semi-simplicity.

Proof. We shall prove that absence of nilpotent elements implies strong regularity; the remaining implications are immediate and are left to the reader to verify. If $A$ is an algebraic algebra without nilpotent elements, the sub-algebra generated by a single element is of finite order and without nilpotent elements, hence is a direct sum of division algebras, that is, strongly regular. If $A$ is a ring with descending chain condition on right ideals and no nilpotent elements, it is a direct sum of division rings and therefore again strongly regular.

(5) In saying that there are no nilpotent elements, we mean of course that there are none other than 0 . In this connection we mention also the following result due to Forsythe and McCoy [5]: a regular ring without nilpotent elements is strongly regular. 
We shall conclude this section with two theorems on strongly regular rings which we shall not need later, but which are perhaps of independent interest.

It is easy to deduce from Theorem 3.2 that the maximal left ideals in a strongly regular ring are two-sided. It is further true that all ideals are twosided. (In the ring of continuous functions from $(0,1)$ to the quaternions, the former statement is true but not the latter, cf. [16, p. 180].)

THEOREM 3.4. In a strongly regular ring all left or right ideals are twosided.

This theorem is an evident consequence of the following lemma.

Lemma. Suppose that $a, b$ are elements in a strongly regular ring $A$ such that $a$ lies in every maximal ideal containing $b$. Then $a=b c$.

Proof. Let $M$ be a maximal ideal. Since $A-M$ is a division ring, there exists an element $c^{*}$ such that $a-b c^{*} \in M$. The set of maximal ideals containing $a-b c^{*}$ includes an open and closed neighborhood $U$ of $M$. If $K$ is the (compact) set where $a$ does not vanish, we can cover $K$ by a finite number of the U's, which we may then replace by disjoint open compact sets, $W_{1}, \cdots, W_{n}$, which precisely cover $K$. For each $W_{i}$, an element like $c^{*}$ is given; we multiply it by the characteristic function of $W_{i}$, obtaining an element $c_{i}$ such that $b c_{i}$ is congruent to $a$ modulo $W_{i}$ and to 0 modulo the complement of $W_{i}$. We take $c=c_{1}+\cdots+c_{n}$.

\section{TheOREM 3.5. A strongly regular Banach algebra is finite-dimensional.}

Proof. Let $M$ be a maximal ideal in the strongly regular Banach algebra $A$. Then $A-M$ is also a Banach algebra and is a division ring. By [17], or [2], $A-M$ is the set of reals, complexes, or quaternions; in any event, an algebra of finite order over the reals. Hence if $a$ is any element in $A$, we can find a polynomial $f$ with real coefficients such that $f(a) \in M$. There will be a neighborhood of $M$ in which $f(a)$ continues to lie; the compact set where $a$ does not vanish is covered by a finite number of these neighborhoods; we take the product of the corresponding polynomials, multiply by $x$, and obtain a polynomial $g(x)$ such that $g(a)$ vanishes at every maximal ideal, that is, $g(a)=0$.

Hence $A$ is an algebraic algebra. It now follows from [15, Theorem 9] that $A$ is finite-dimensional.

4. Remarks on commutativity. In the sequel we shall be discussing commutative algebraic algebras for the most part. However in some cases the assumption of commutativity is redundant. Such a result has been proved by Jacobson [13, Theorem 9]. Following his method it is possible to prove a more general theorem. 
THEOREM 4.1. Let $K$ be a field with the following property: any division algebra of finite order over $K$ is commutative, and if it is separable over $K$ it is normal. Then any algebraic algebra $A$ over $K$ without nilpotent elements is commutative.

Proof. Since $A$ is strongly regular (Theorem 3.3) it suffices to prove the theorem for the case where $A$ is a division algebra. Let $C$ be the center of $A$; we suppose $A \neq C$. The argument of $[13$, Lemma 2] shows that $A$ contains an element $x \in C$ which is separable over $C$. Our hypothesis implies that all the conjugates of $x$ over $K$ are polynomials in $x$ with coefficients in $K$. It follows $a$ fortiori that $C(x)$ is normal over $C$, and therefore admits a nontrivial automorphism over $C$ that sends $x$ into a polynomial $f(x)=\sum a_{i} x^{i}$ with coefficients $a_{i}$ in $C$. By [3, Theorem 12] this automorphism can be extended to an inner automorphism of $A$, say by the element $y$, so that $y^{-1} x y=f(x)$. The algebra $K\left(x, y, a_{i}\right)$ can now be seen to be of finite order over $K$, yet it is noncommutative, contrary to hypothesis.

As a fairly direct consequence of Theorem 4.1 (or of Jacobson's more special result), one can show [13, Theorem 11] that a ring in which every element satisfies $a^{n(a)}=a, n(a)>1$, is commutative. This result can be extended to a class of rings studied by McCoy [19].

THEOREM 4.2. Let $A$ be a ring without nilpotent elements, and such that every element $a \neq 0$ has finite additive order and satisfies a polynomial equation with integral coefficients

$$
r_{m} a^{m}+\cdots+r_{1} a=0 \quad\left(r_{m} a^{m} \neq 0\right) .
$$

Then every element satisfies an equation $a^{n(a)}=a, n(a)>1$, and so $A$ is commutative.

Proof. First suppose $a$ has prime additive order, $p a=0$. Then the polynomial equation for $a$ can be written with highest coefficient unity. Hence the subring $S$ generated by $a$ is finite and without nilpotent elements, and so is a direct sum of finite fields. The existence of an equation $a^{n(a)}=a$ follows.

Now take a general element $a$ of additive order $k$. From the absence of nilpotent elements, it follows that $k$ is a product $p_{1} \cdots p_{j}$ of distinct primes. The subring generated by $a$ is then a direct sum of rings of characteristics $p_{1}, \cdots, p_{j}$. From the preceding result we deduce $a^{n(a)}=a$.

5. Preliminary representation theorems. We begin this section with a fairly immediate consequence of Theorem 2.3.

THEOREM 5.1. Let $A$ be an algebraic algebra without nilpotent elements over a field $K$; suppose that every element $a$ in $A$ satisfies an equation $f(a)=0$, where $f(x)$ is a polynomial with coefficients in $K$ which factors completely in $K$. Then $A$ is isomorphic to the set of all continuous functions, vanishing outside a compact set, from a locally compact zero-dimensional space to $K$. 
Proof. $A$ is strongly regular by Theorem 3.3. If $M$ is a maximal ideal in $A$, our hypotheses imply that the natural map of $K$ into $A-M$ is an isomorphism of $K$ and $A-M$. Theorem 5.1 then follows from Theorem 2.3.

COROLlary 1. An algebraic algebra without nilpotent elements over an algebraically closed field $K$ is isomorphic to the set of all continuous functions, vanishing outside a compact set, from a locally compact zero-dimensional space to $K$.

The next corollary generalizes Stone's topological representation of Boolean rings, and also subsumes a theorem of McCoy and Montgomery [18].

CoROLlaRy 2. Let $A$ be an algebra over $G F\left(p^{n}\right)$ in which every element satisfies the equation $a^{p^{n}}=a$. Then $A$ is isomorphic to the set of all continuous functions, vanishing outside a compact set, from a locally compact zero-dimensional space to $G F\left(p^{n}\right)$.

The following further generalization is also an immediate consequence of Theorem 5.1.

Corollary 3. Let $K$ be an absolutely algebraic field of characteristic $p$, and let $A$ be an algebraic algebra over $K$ such that every element satisfies an equation $a^{p^{n}}=a$, where $n$ is a divisor of the G-number of $K$. Then $A$ is isomorphic to the set of all continuous functions, vanishing outside a compact set, from a locally compact zero-dimensional space to $K$.

The hypothesis of Theorem 5.1 has the effect of making all residue fields isomorphic to $K$, and thus allowing the use of Theorem 2.3. We shall now explore two relaxations of this hypothesis. The first of these still demands that all residue fields be isomorphic, but they are now allowed to be isomorphic to a proper extension of $K$.

THEOREM 5.2. Let $L$ be a division algebra of finite order over a field $K$, and suppose that $A$ is an algebraic algebra without nilpotent elements over $K$, possessing a unit element, and such that for every maximal ideal $M, A-M$ and $L$ are isomorphic (as algebras over $K$ ). Then $A$ is isomorphic to the set of all continuous functions from a compact zero-dimensional space to $L$.

Proof. Let $u_{1}, \cdots, u_{n}$ be a basis of $L$ over $K$, with $u_{i} u_{j}=\sum \alpha_{i j k} u_{k}$ $\left(\alpha_{i j k} \in L\right)$. For any maximal ideal $M$ we pick elements $v_{i}$ mapping on $u_{i}$ modulo $M$. Then

$$
v_{i} v_{j}-\sum \alpha_{i j k} v_{k} \in M
$$

For each $i, j$ this extends to an open and closed neighborhood of $M$, and by taking the intersection of these $n^{2}$ neighborhoods, we obtain an open and closed neighborhood $U$. such that $v_{i} v_{j}$ is congruent to $\sum \alpha_{i j k} v_{k}$ for every $i, j$ and every maximal ideal in $U$. Since $A$ has a unit element, its structure space 
$S$ is compact, and is therefore covered by a finite number of the $U$ 's. These may then be decomposed further to disjoint open and closed sets covering $S$. We multiply the $v$ 's corresponding to a given one of these sets by its characteristic function; we add the modified $v$ 's, obtaining say $w_{1}, \cdots, w_{n}$. Then $w_{i} w_{j}=\sum \alpha_{i j k} w_{k}$, and the subalgebra of $A$ generated by $w_{1}, \cdots, w_{n}$ is isomorphic to $L$ and may be identified with $L$. We may now regard $L$ as being a set of left operators on $A$, and it is clear that for any maximal ideal $M$, the natural map of $L$ into $A-M$ is an isomorphism. The conclusion now follows from Theorem 2.3.

Remark. Since Theorem 5.2 gives a complete solution of the structure problem whenever it is applicable, it would be of interest to know whether its conclusion is obtainable under weaker hypotheses. Whether the hypothesis that $L$ is finite-dimensional is necessary we have not been able to determine. However the following two hypotheses cannot be dropped: that $A$ has a unit element, and that $L$ is algebraic over $K$. Indeed if either of these assumptions is omitted, $A$ may simply fail to admit $L$ as a set of operators at all. An example for the case where $A$ has no unit will be given at the end of $\S 8$. An example for the case where $L$ is transcendental over $K$ is given in the following paragraph.

Let $R$ denote the field of rational numbers, and $R(x)$ a simple transcendental extension. Let $A$ be the set of all sequences with a "limit element": $\left(a_{1}, a_{2}, \cdots, a_{n}, \cdots a\right)$ where $a_{n}, a \in R(x)$, and $\dot{a_{n}}=a(n)$ from some point on. In greater detail: if $a$ is a rational number, than $a_{n}=a$ from some point on, and otherwise $a_{n}$ is from some point on the rational function $a$ evaluated at $x=n$. It can easily be verified that a maximal ideal in $A$ consists precisely of all sequences having 0 at a designated place, and thus every residue field is isomorphic to $R(x)$. However, since every sequence consists of rational numbers from some point on, it is impossible for $A$ to admit $R(x)$ as a field of operators.

The Theorem 5.3 will now be proved as a preliminary to the results of $\S 6$. It asserts in effect that purely inseparable extensions cause no difficulty.

THEOREM 5.3. Let $A$ be an algebraic algebra without nilpotent elements over a separably algebraically closed field $K$. Let $L$ be the algebraic closure of $K,\left\{K_{\alpha}\right\}$ the totality of fields between $K$ and $L$. Then there exists a locally compact zerodimensional space $X$ with a system of closed subsets $\left\{X_{\alpha}\right\}$, for which we can make the following statement: $A$ is isomorphic to the set of all continuous functions from $X$ to $L$, which vanish outside a compact set, and in $X_{\alpha}$ take values in $K_{\alpha}$.

Proof. We need only treat explicitly the case of characteristic $p$, for Theorem 5.3 and Theorem 5.1, Corollary 1 coincide for characteristic 0 . We observe that, by Theorem $4.1, A$ is commutative. For any maximal ideal $M$, $A-M$ is thus isomorphic to a field between $K$ and $L$, and, since $p$ th roots are 
unique, there is precisely one such isomorphic embedding in $L$. Let $X_{\alpha}$ denote the set of maximal ideals for which the residue field is $K_{\alpha}$ or a subfield of $K_{\alpha}$. We assert that $X_{\alpha}$ is closed (in the structure space $X$ ). For let $M$ be any maximal ideal in the complement of $X_{\alpha}$. Some element in $A$ must take at $M$ a value not in $K_{\alpha}$. It continues to take the same value in a neighborhood of $M$. Hence the complement of $X_{\alpha}$ is open.

By repetition of the arguments used in the latter half of the proof of Theorem 2.3, we represent $A$ as a set of continuous functions from $X$ to $L$, each vanishing outside a compact set. On $X_{\alpha}$ the values necessarily lie in $K_{\alpha}$. It only remains to be shown that we indeed get all continuous functions $f$ with $f\left(X_{\alpha}\right) \subset K_{\alpha}$. To see this, take any maximal ideal $M$, where the residue field $A-M$ is, say, $K^{\prime}$. Then $M$ lies in the set associated with $K^{\prime}$, and $f$ necessarily takes a value in $K^{\prime}$ at $M$. There exists in $A$ an element $a$ taking this same value at $M$. The coincidence of values of $a$ and $f$ extends to an open and closed neighborhood of $M$, By an argument already used several times $\left(^{6}\right)$, we build an element in $A$ coinciding everywhere with $f$.

6. The structure of algebraic algebras. By a further refinement of Theorem 5.3 we are able to obtain a reasonably clear insight into the structure of an arbitrary commutative semi-simple algebraic algebra $A$ over a field $K$. The procedure is as follows: we form over $K$ the Kronecker product (also called the direct product) of $A$ and the separable algebraic closure $L$ of $K$, written $A \times L$. Then $A \times L$ is an algebra over $L$, and its structure is known from Theorem 5.3. It remains to describe some way of characterizing $A$ in $A \times L$, and it will appear that this can be done by using the automorphisms of $L$ over $K$. The detailed result is given in the following theorem.

THEOREM 6.1. Let $A$ be a commutative semi-simple algebraic algebra over a field $K$; let $L$ be $\left(^{7}\right)$ the separable algebraic closure of $K$, and $M$ the algebraic closure of $K$. Let $G$ be the Galois group of $L / K$; let $\left\{L_{\alpha}\right\}$ denote the set of subfields of $M$ which contain $L$. Then there exists a locally compact zero-dimensional space $X$ with a set $\left\{X_{\alpha}\right\}$ of closed subsets, and a representation of $G$ by a group $G^{*}$ of homeomorphisms of $X$, such that we can assert: $A$ is isomorphic to the set of all continuous functions $f$ from $X$ to $M$ satisfying (1) each $f$ vanishes outside some compact set, (2) $f\left(X_{\alpha}\right) \subset L_{\alpha}$, (3) $f\left[g^{*}(x)\right]=g[f(x)]$ for any $x \in X$ and $g \in G$.

Proof. The Kronecker product $A \times L$ is a commutative algebraic algebra

(6) The argument we are referring to runs as follows: obtain a finite covering by open and closed sets, reduce it to a disjoint covering, and then make use of the characteristic functions (cf. the proofs of Theorems 2.3, 3.4, and 5.2). Despite the obvious repetitive nature of the instances of this argument, we have not been able to formulate a lemma covering them all.

(7) Actually ( $L) M$ need not be taken to be the (separable) algebraic closure of $K$; it would suffice to take fields large enough to cover the actual residue fields which occur in $A$. A similar remark applies to Theorem 5.3. We shall exploit this economy to a certain extent later in $\$ 8$; but we have refrained from mentioning it in the statement of Theorem 6.1, which is sufficiently complicated already. 
over $L$. Moreover it is semi-simple. To prove this, we take any element $\sum a_{i} m_{i}$ in $A \times L\left(a_{i} \in A, m_{i} \in L\right)$, and let $B$ denote the subalgebra of $A$ generated by the $a$ 's. Then $B$ is a direct sum of fields, and by $[18$, Theorem $3, \mathrm{p}$. 96], $B \times L$ is semi-simple, that is, has no nilpotent elements. Hence $A \times L$ has no nilpotent elements.

Now we apply Theorem 5.3 to $A \times L$, thus representing it as the set of all continuous functions from $X$ to $M$ with $f\left(X_{\alpha}\right) \subset L_{\alpha}$, where $X$ is the structure space of $A \times L$, and so on. Let $g$ be any automorphism of $L$ over $K$ (note that it extends uniquely to an automorphism of $M$ over $K$ ). The functional representation allows us to define in a natural way an analogous automorphism of $A \times L$, which we shall again write as $g$ : we send $f$ into $f_{1}=g(f)$ where $f_{1}(x)=\dot{g}[f(x)]$ for every $x \in X$.

There is another way of allowing $g$ to induce an automorphism of $A \times L$, and we shall write $g^{\prime}$ for this second automorphism. Choose a fixed Hamel basis $\left\{m_{i}\right\}$ of $L$ over $K$, and if $a=\sum a_{i} m_{i}$ define $g^{\prime}(a)=\sum a_{i} g\left(m_{i}\right)$. This mapping is independent of the choice of basis: suppose $\left\{n_{j}\right\}$ is a second basis, where $a=\sum b_{j} n_{j}$, and $m_{i}=\sum \theta_{i j} n_{j}\left(\theta_{i j} \in K\right)$. From known properties of the Kronecker product, we infer $b_{j}=\sum \theta_{i j} a_{i}$. Then

$$
\sum b_{j} g\left(n_{j}\right)=\sum \theta_{i j} a_{i} g\left(n_{j}\right)=\sum a_{i} g\left(m_{i}\right) .
$$

Next we show that $g^{\prime}$ is an automorphism of $A \times L$. That it preserves addition and multiplication follows readily from the fact that $g$ does. The kernel of $g^{\prime}$ is 0 , for if $0=g^{\prime}(a)=\sum a_{i} g\left(m_{i}\right)$ it follows from the fact that $\left\{g\left(m_{i}\right)\right\}$ is a Hamel basis of $L / K$ that $a_{i}=0, a=0$. Finally $g^{\prime}$ has all of $A \times L$ for its image; in fact, the element $\sum a_{i} g^{-1}\left(m_{i}\right)$ is sent into $\sum a_{i} m_{i}$.

We assert $\left({ }^{8}\right)$ that the elements left invariant by every $g^{\prime}$ comprise precisely $A$. That elements of $A$ are left fixed is evident from the invariance of the definition of $g^{\prime}$, and from the fact that we may take unity for one of the elements of the basis of $L$ over $K$. Conversely, suppose that $\sum a_{i} m_{i}=\sum a_{i} g\left(m_{i}\right)$ for every g. Write $a_{i}=\sum \lambda_{i j} c_{j}, \lambda_{i j} \in K$, where the $c_{j} \in A$ are linearly independent over $K$. Then $\sum \lambda_{i j} c_{j}\left[m_{i}-g\left(m_{i}\right)\right]=0$. Hence $\sum \lambda_{i j}\left[m_{i}-g\left(m_{i}\right)\right]$ vanishes for each $j$. This says that $\sum \lambda_{i j} m_{i}$ is invariant under every automorphism of $L / K$, whence it must lie in $K$. It follows (if we take $m_{1}=1$ ) that $\lambda_{i j}=0$ for $i \geqq 2$. Hence $\sum a_{i} m_{i}=a_{1} \in A$, as desired.

The automorphism $\left(g^{\prime}\right)^{-1} g$ sends $\sum a_{i} m_{i}$ into $\sum g\left(a_{i}\right) m_{i}$, and is therefore an $L$-automorphism of $A \times L$. Now any $L$-automorphism of $A \times L$ induces a homeomorphism of the structure space $X$, since it permutes the maximal ideals and preserves the (algebraically defined) topology. We write $g^{*}$ for the homeomorphism induced by $\left(g^{\prime}\right)^{-1} g$. This mapping is clearly a homeo-

(8) A similar fact can be proved in a more general context. Let $A, B$ be algebras over $K$. and $G$ a group of $K$-automorphisms of $B$ having $B_{0}$ as the sub-algebra fixed under all automorphisms of $G$. We define automorphisms in $A \times B$ by applying those in $G$ to the $B$-component. Then the fixed sub-algebra is $A \times B_{0}$. 
morphism of $G$ onto a group $G^{*}$ of homeomorphisms of $X$. The statement $f\left[g^{*}(x)\right]=g[f(x)]$ for all $x \in X$ is equivalent to the assertion that $\left(g^{\prime}\right)^{-1} g$ and $g$ are identical on $f$, that is, $f$ is invariant under $g^{\prime}$. The set for which this is true for all $g$ comprises precisely $A$, as we showed above. This concludes the proof of Theorem 6.1.

It is perhaps worth restating Theorem 6.1 in the considerably simplified form that it takes when $K$ is real closed.

COROLlARY. Let $A$ be a commutative semi-simple algebraic algebra over a real closed field $K$. Then there exists a locally compact zero-dimensional space $X$ with a homeomorphism $\sigma$ whose square is the identity, for which we can make the following statement: $A$ is isomorphic to the set of all continuous functions from $X$ to $K(i)$, which vanish outside a compact set, and for all $x \in X$ take conjugate values at $x$ and $\sigma(x)$.

Let $A$ be a ring in which every element satisfies an equation $a^{m}=a(m$ independent of $a, m>1$ ). We can give a fairly complete result on the structure of $A$. First, $A$ is a direct sum of rings of prime characteristic $\left({ }^{9}\right)$; hence it suffices to take $A$ of characteristic $p$. Then the residue fields are all of the form $G F\left(p^{k}\right)$ with $\left(p^{k}-1\right) \mid(m-1)$. Let $n$ be the L.C.M. of all the $k$ 's; then $a^{p^{n}}=a$ for all $a \in A$. We now apply the procedure of Theorem 6.1 with the modification that we take the Kronecker product of $A$ with $G F\left(p^{n}\right)$ rather than with the algebraic closure of $G F(p)$ (cf. footnote 7). Since the Galois group of $G F\left(p^{n}\right)$ over $G F(p)$ is cyclic of order $n$, the result can be stated as follows.

THEOREM 6.2. Let $A$ be a ring of characteristic $p$, that is, an algebra over $G F(p)$, such that each element satisfies $a^{p^{n}}=a$ for a fixed $n$. There exists a locally compact zero-dimensional space $X$ with a homeomorphism $\sigma$ whose nth power is the identity, for which we can make the following statement: $A$ is isomorphic to the set of all continuous functions from $X$ to $G F\left(p^{n}\right)$, which vanish outside a compact set, and satisfy $f(\sigma x)=[f(x)]^{p}$ for every $x \in X$.

A similar discussion can be given for rings satisfying $a^{m(a)}=a$. However the resulting theorem (after reduction to prime characteristic) is simpler than Theorem 6.1 only through the absence of inseparable extensions, and so we shall not state it explicitly.

Further representations for more special algebras will be proved in $\S 8$.

7. Solvable groups of homeomorphisms. The structure theorems of the preceding section have led to the consideration of a space $X$ with a group of homeomorphisms $G$, and a certain class of functions on $X$. The restriction on the functions may be described as follows: the value of a function at a point $x \in X$ determines its values on the entire orbit of $x$ (the orbit of $x$ being the

(9) The eligible primes are precisely those for which $p-1$ divides $m-1$. 
totality of points $g(x), g \in G)$. From this point of view it is natural to seek a representation on a smaller space by selecting a single representative from each orbit. Of course in doing so, we must pay our respects to the topology of $X$. We formulate the following definition.

Definition. A fundamental domain for a group of homeomorphisms $G$ on a space $X$ is a subset $X_{a}$, containing exactly one point from each orbit, and such that the natural mapping $X \rightarrow X_{G}$ is continuous.

It is evident that a fundamental domain is necessarily closed. The converse is not true, as shown by the following example: let $X$ be the set of all integers with a limit point $\infty(=-\infty)$ adjoined, and $G$ the infinite cyclic group generated by the homeomorphism $i \rightarrow i+1, \infty \rightarrow \infty$. The subset $(0, \infty)$ is closed and contains just one point from each orbit, but it is not a fundamental domain; indeed no fundamental domain exists. However for finite groups the converse does hold.

LEMMA 7.1. Let $G$ be a finite group of homeomorphisms of a space $X$. The set $X_{G}$ is a fundamental domain if and only if it is closed in $X$ and contains $j u s t$ one point from each orbit.

Proof. We have only to prove the continuity of $X \rightarrow X_{G}$ under the stated assumptions. Let us write $F(x)$ for the $X_{G}$-representative of $x$. Suppose the directed set $\left\{x_{\alpha}\right\}$ converges to $x$, but that $\left\{F\left(x_{\alpha}\right)\right\}$ fails to converge to $F(x)$. There will exist a neighborhood $U$ of $F(x)$ such that a cofinal subset $\left\{F\left(x_{\beta}\right)\right\}$ of $\left\{F\left(x_{\alpha}\right)\right\}$ lies outside $U$. For each $\beta, F\left(x_{\beta}\right)=g_{\beta}\left(x_{\beta}\right)$ for some $g_{\beta} \in G$. Since $G$ is finite, there will exist an element $g$ in $G$ and a cofinal subset $\left\{x_{\gamma}\right\}$ such that $F\left(x_{\gamma}\right)=g\left(x_{\gamma}\right)$. Now $g\left(x_{\gamma}\right) \rightarrow g(x)$, and since $X_{G}$ is closed, $g(x) \in X_{G}$. Hence $g(x)=F(x)$, a contradiction since $g(x)$ lies outside $U$.

Suppose the group $G$ of homeomorphisms of $X$ has a subgroup $H$. Then $H$ may itself be regarded as a group of homeomorphisms of $X$. We suppose that a fundamental domain $X_{H}$ exists. Then if $H g$ is a right coset of $H$, there is a natural way of using it to induce a continuous mapping of $X_{H}$ onto itself, namely, we send $x \in X_{H}$ into the $X_{H}$-representative of $g(x)$. That this definition is unique follows from the fact that for $h \in H, g(x)$ and $h g(x)$ lie in the same orbit under $H$. Moreover the mapping is continuous, for it is the product of the continuous mappings $x \rightarrow g(x)$ and $X \rightarrow X_{H}$. Finally the mapping is clearly one-to-one on all of $X_{H}$.

In the event that $H$ is a normal subgroup, each element of $G / H$ induces a homeomorphism of $X_{H}$, since we may apply the above argument to the inverse mapping. Thus we may regard $G / H$ as a group of homeomorphisms of $X_{H}$. Suppose that again a fundamental domain $\left(X_{H}\right)_{G / H}$ exists. The mapping $X \rightarrow\left(X_{H}\right)_{G / H}$ is continuous, being the product of two continuous mappings, and it is easy to verify that $\left(X_{H}\right)_{G / H}$ contains exactly one point from each orbit of $X$ under $G$. This remark shows that for finite groups (or more generally for groups with a finite composition series), the problem of constructing a funda- 
mental domain need only be considered for the case of simple groups. We summarize in the following lemma.

LemMA 7.2. Let $G$ be a group of homeomorphisms of a space $X, H$ a normal subgroup, $Y_{H}$ a fundamental domain for $X$ under $H$, and $X_{G}=\left(X_{H}\right)_{G / H} a$ fundamental domain for $X_{H}$ under $G / H\left(G / H\right.$ acting on $X_{H}$ as described above). Then $X_{G}$ is a fundamental domain for $X$ under $G$.

We now turn our attention to the actual construction of a fundamental domain $X_{G}$. We are able to effect the construction with the aid of two hypotheses, one purely algebraic, the other purely topological: (1) $G$ is a finite solvable group, (2) every open set in $X$ can be expressed as a union of disjoint open compact sets. Hypothesis (2) obviously implies that $X$ is locally compact, and moreover, if $X$ is assumed to be a Hausdorff space (so that compact sets are closed), it also implies zero-dimensionality. In the applications to follow in $\S 8, X$ will indeed be locally compact and zero-dimensional, so that these restrictions are acceptable $\left({ }^{10}\right)$.

We note two case where hypothesis (2) is satisfied: (a) $X$ is a discrete space, (b) $X$ is a locally compact zero-dimensional space satisfying the second axiom of countability. The latter statement is a consequence of the following result.

LEMMA 7.3. If an open set $W$ is the union of a countable number of open compact sets, then $W$ is the union of a countable number of disjoint open compact sets.

Proof. If $W=U_{1} \cup U_{2} \cup \ldots$ with $U_{i}$ compact open, then also $W=V_{1}$ $\cup V_{2} \cup \ldots$ where $V_{i}=U_{i}-\left(U_{1} \cup U_{2} \cup \ldots \cup U_{i-1}\right)$. The $V$ 's are disjoint open compact sets.

We now prove the main result of this section.

THEOREM 7.1. Let $X$ be a locally compact zero-dimensional space with the property that any open set in $X$ is a union of disjoint compact open sets. Let $G$ be a finite solvable group of homeomorphisms of $X$. Then a fundamental domain $X_{G}$ exists.

Proof. We first remark that our hypothesis on $X$, that any open set is a union of disjoint open compact sets, is inherited by any closed subset of $X$. It then follows from Lemma 7.2 that it will suffice to consider the case where $G$ is cyclic of prime order $p$, say with generating element $g$. Let $X_{0}$ be the (closed) set of fixed points. We represent $X-X_{0}$ as a union of disjoint open compact sets $\left\{U_{\alpha}\right\}$. For each point $x$ in $U_{\alpha}$ we pick an open compact neighbor-

(10) Theorem 7.1 could be proved under the following weaker hypothesis: every open set in $X$ is a union of disjoint open and closed sets, each of which has the property that a covering by open sets can be reduced to a countable covering. But in locally compact spaces this assumption is equivalent to hypothesis (2), and so we have omitted this refinement. 
hood $V$ contained in $U_{\alpha}$, and such that $V, g V, \cdots, g^{p-1} V$ are all disjoint; $U_{\alpha}$ is covered by a finite number of the $V$ 's, and these may be decomposed further to disjoint open compact sets. We well order the totality of sets thus obtained and, changing notation, we have that $X-X_{0}$ is the union of disjoint open compact sets $\left\{V_{\alpha}\right\}$, each of which is disjoint from its images under $G$. Define $W_{1}=V_{1}$, and assuming by induction that $W_{\alpha}$ is defined for $\alpha<\lambda$, set

$$
\dot{Y}_{\lambda}=\bigcup_{\alpha<\lambda} W_{\alpha}, \quad W_{\lambda}=V_{\lambda}-\left(Y_{\lambda} \cup g Y_{\lambda} \cup \cdots \cup g^{p-1} Y_{\lambda}\right) .
$$

We further assume by induction $W_{\alpha}$ is open and closed for $\alpha<\lambda$. Then $Y_{\lambda}$ is open, as is $g^{i} Y_{\lambda}$, whence $W_{\lambda}$ is closed. Next we remark that $Y_{\lambda} \cup X_{0}$ is closed. For suppose $x$ is in its complement $Z$; then $x$ is in some $V_{\beta}$. If $\beta \geqq \lambda$, we have $V_{\beta} \subset Z$, and if $\beta<\lambda$ then $x$ is not in $W_{\beta}$ and, since $W_{\beta}$ is closed, a neighborhood of $x$ is in $Z$. Thus $Z$ is open, and $Y_{\lambda} \cup X_{0}$ is closed, as is $g^{i} Y_{\lambda} \cup X_{0}$. From this it follows that $W_{\lambda}$ is open.

Let $Y$ denote the union of all the $W$ 's. It is clear that $Y, g Y, \cdots, g^{p-1} Y$ are disjoint and constitute a covering of $X-X_{0}$. The set $Y \cup X_{0}$ is closed, by a repetition of the argument given above for $Y_{\lambda} \cup X_{0}$. Hence $Y \cup X_{0}$ is the desired fundamental domain $X_{G}$.

Remark. We have not been able to determine whether the hypothesis that $G$ is solvable is necessary in Theorem 7.1. However some kind of topological hypothesis, going beyond zero-dimensionality and local compactness or even compactness, is indispensable, as is shown by the following example( $\left.{ }^{11}\right)$.

Take the set of all ordinals up to and including $\omega$, and augment it by placing after $\omega$ the finite ordinals in reverse order:

$$
X_{\omega}: 1,2, \cdots, n, \cdots, \omega, \cdots, n^{*}, \cdots, 2^{*}, 1^{*} .
$$

With open intervals as a basis for open sets, $X_{\omega}$ is a compact zero-dimensional space. We proceed in a similar manner with the first uncountable ordinal $\Omega$,

$X_{\Omega}: 1,2, \cdots, \omega, \cdots, \alpha, \cdots, \Omega, \cdots, \alpha^{*}, \cdots, \omega^{*}, \cdots, 2^{*}, 1^{*}$.

The Cartesian product $X=X_{\omega} \times X_{\Omega}$ is the desired space; it is compact and zero-dimensional. The correspondences $n \leftrightarrow n^{*}, \omega \leftrightarrow \omega$ in $X_{\omega}$ and $\alpha \leftrightarrow \alpha^{*}$, $\Omega \leftrightarrow \Omega$ in $X_{\Omega}$ induce a homeomorphism $\theta$ of order 2 in $X$, in which $(n, \alpha)$ $\leftrightarrow\left(n^{*}, \alpha^{*}\right),\left(n^{*}, \alpha\right) \leftrightarrow\left(n, \alpha^{*}\right)$, and so on. The only fixed point is $(\omega, \Omega)$, and it is noteworthy that the open set $X-(\omega, \Omega)$ cannot be expressed as a union of disjoint open and closed sets, so that at least the method of proof of Theorem 7.1 fails. Let $G$ be the group of order two generated by $\theta$. We assert that no fundamental domain $X_{G}$ can exist. For $X_{G}$ would have to contain infinitely many of either

$$
(1, \Omega), \cdots,(n, \Omega), \cdots
$$

(11) We are greatly indebted to J. L. Kelley for providing us with this example. 
or

$$
\left(1^{*}, \Omega\right), \cdots,\left(n^{*}, \Omega\right), \cdots .
$$

We may suppose that the former is the case, so that $\left(n_{k}, \Omega\right) \in X_{G}$, where $n_{k} \rightarrow \infty$ as $k \rightarrow \infty$. Along with $\left(n_{k}, \Omega\right), X_{a}$ must also contain an entire neighborhood consisting, say, of all $\left(n_{k}, y\right)$ with $y$ between $\alpha_{k}$ and $\alpha_{k}^{*}\left(\alpha_{k}<\Omega\right)$. Let $\alpha=\lim \sup \alpha_{k}$; clearly $\alpha<\Omega$. Then $\left(n_{k}, \alpha\right)$ and $\left(n_{k}, \alpha^{*}\right)$ lie in $X_{G}$ for all $k$, whence $(\omega, \alpha)$ and $\left(\omega, \alpha^{*}\right)$ are both in $X_{G}$ since the latter is closed. This contradicts $\theta(\omega, \alpha)=\left(\omega, \alpha^{*}\right)$, and concludes the proof that no fundamental domain exists.

8. Functions on structure spaces. In $\S 6$ we developed representations of an algebra $A$ over a field $K$ as a class $A^{\prime}$ of certain functions on a topological space $X$ to the algebraic closure $M$ of $K$, the class $A^{\prime}$ being defined by two kinds of conditions. The first is topological : each $f$ is to be continuous, vanish outside a compact set, and its values are restricted to subfields of $M$ on certain closed subsets of $X$. The second is algebraic: there is a representation $g^{*}$ of each $g$ in the Galois group of $M / K$ as a homeomorphism of $X$, and each $f$ is to satisf $f\left[g^{*}(x)\right]=g[f(x)]$.

The purpose of this section is to show how the results of $\$ 7$ can be applied to reduce representations of the type described above to simpler ones in which all the restrictions are of the first kind. The essence of the reduction is this: we regard our functions as being defined merely on the fundamental domain, and ignore the rest of the space. Since we have seen that a fundamental domain does not always exist, it is natural to expect that restrictive assumptions must be imposed, and at the end of this section we give an example showing the necessity of some such restriction. We shall assume that every ideal $I$ in $A$ is countably generated, that is, $I$ contains a countable set $S$ such that $I$ is the smallest ideal containing $S$. Any algebra of countable order of course fulfills this condition. While weaker.assumptions would suffice, it does not seem to be possible to state these neatly directly in terms of $A$, rather than in terms of its structure space.

This section can also be regarded as giving sufficient conditions that a ring may be represented as a ring of continuous functions on its own structure space; for it is easy to verify that the space $X$ of Theorem 8.1 is precisely the structure space of $A$, while that of Theorem 6.1 is in general a larger space.

We first prove some preliminary results.

Lemma 8.1. Let $A$ be a biregular ring in which every two-sided ideal is countably generated. Then every open set $W$ in the structure space $X$ of $A$ is the union of a countable number of disjoint open compact sets.

Proof. Let $I$ be the intersection of the maximal ideals which comprise $X-W$, suppose $I=\left(a_{i}\right)$, and let $W_{i}$ be the set of maximal ideals not containing $a_{i}$. By Lemma $1.1, W_{i}$ is an open compact set, and it is evident that 
$W_{i} \subset W$. For any $M$ in $W$ we cannot have $I \subset M$, for this implies $M \in X-W$ since the latter is closed. Hence $a_{i} \notin M$ for some $i$, and $M \in W_{i}$. It follows that $W=U W_{i}$ and, by Lemma 7.3, $W$ is the union of a countable number of disjoint open compact sets.

Lemma 8.2. Let $A$ be an algebra over $K$ such that every (left) ideal in $A$ is countably generated; let $L$ be an algebra of finite order over $K$ with a unit element, and $B$ the Kronecker product $A \times L$ over $K$. Then any (left) ideal in $B$ is countably generated.

Proof. Choose a fixed basis $m_{1}, \cdots, m_{n}$ of $L / K$. Any element in $B$ then has a unique representation $a_{1} m_{1}+\cdots+a_{n} m_{n}\left(a_{r} \in A\right)$. Now let $I$ be any left ideal in $B$. For $r=1, \cdots, n$ define $I_{r}$ to be the set of all elements of $I$ of the form $a_{1} m_{1}+\cdots+a_{r} m_{r}$. Since $A \subset B$, the set of coordinates $a_{r}$ which occur in elements of $I_{r}$ forms a (left) ideal $J_{r}$ in $A$. Let $J_{r}=\left(b_{i r}\right)$, and let $c_{i r}$ denote an element in $I_{r}$ having $b_{i r}$ as its $r$ th coordinate. Then the elements $c_{i r}(r=1, \cdots, n, i=1,2, \cdots)$ form a basis for $I$ in $B$.

We shall now prove our main result.

THEOREM 8.1. Let $A$ be a commutative semi-simple algebraic algebra over a field $K$, suppose that all residue fields of $A$ are isomorphic (as algebras over $K$ ) to subfields of a certain field $L$ which is finite, normal, and solvable over $K$, and let $\left\{L_{i}\right\}$ denote the set of fields between $K$ and $L$. Suppose that every ideal in $A$ is countably generated. Then there exists a zero-dimensional locally compact space $X$, with a set of closed subsets $\left\{X_{i}\right\}$, for which we can make the following statement: $A$ is isomorphic to the set of all continuous functions from $X$ to $L$ which vanish outside a compact set and on $X_{i}$ take values in $L_{i}$.

Proof. We apply Theorem 6.1, but with the proviso that the Kronecker product is taken with $L$ rather than the separable algebraic closure of $K$ (cf. footnote 7). Also we note the simplification made possible by our assumption that $L / K$ is separable, as we have done to avoid complications, but at the price of some generality. Thus we have a locally compact zero-dimensional space $Y$ (the structure space of $A \times L$ ) and a representation $G^{*}$ of the Galois group $G$ of $L / K$ by homeomorphisms of $Y ; A$ is isomorphic to the set of all continuous functions from $Y$ to $L$, vanishing outside a compact set, and such that

$$
f\left[g^{*}(y)\right]=g[f(y)] \quad(y \in Y, g \in G) .
$$

By Lemmas 8.1 and 8.2 we know that $Y$ satisfies the hypothesis of Theorem 7.1 ; also $G^{*}$ (being a homomorphic image of $G$ ) is solvable. Hence $Y$ possesses a fundamental domain, say $X$, with respect to $G^{*}$. For any field $L_{i}$ between $K$ and $L$, let $G_{i}$ denote the corresponding subgroup of $G, Y_{i}$ the set of points of $Y$ left fixed by $G_{i}{ }^{*}$, and set $X_{i}=Y_{i} \cap X$. Evidently if $f \in A \times L$ is one of the original elements of $A$ we have $f(y) \in L_{i}$ for $y \in Y_{i}$. We wish to show that, 
conversely, every continuous function from $X$ to $L$, satisfying

$$
f(x) \in L_{i} \quad\left(x \in X_{i}\right),
$$

can be extended to a continuous function from $Y$ to $L$ satisfying (1); with this the proof of Theorem 8.1 will be complete.

Suppose then that $f$ is a continuous function from $X$ to $L$ satisfying (2). For $y \in Y$ we define

$$
f(y)=g[f(x)],
$$

where $g$ is selected so that $g^{*}$ is a homeomorphism for which $y=g^{*}(x)$. This $g$ is not unique, but if we let $G_{i}{ }^{*}$ denote the subgroup leaving $x$ fixed, then $g$ is unique modulo $G_{i}$. Since $x \in X_{i}$, reference to (2) shows that (3) is a consistent definition. Evidently (1) will hold for the extended $f$. It remains only to prove its continuity on $Y$. Suppose on the contrary that we have a directed set $\left\{y_{\alpha}\right\}$ converging to $y$ in $Y$, but such that $f\left(y_{\alpha}\right) \neq f(y)$. After passage to a cofinal subset, we may assume that for a fixed $g \in G, y_{\alpha}=g^{*}\left(x_{\alpha}\right)$ with $x_{\alpha} \in X$. Since $\left(g^{*}\right)^{-1}$ is continuous, we have also $y=g^{*}(x), x \in X$. Thus (3) holds and also $f\left(y_{\alpha}\right)=g\left[f\left(x_{\alpha}\right)\right]$. Since $f$ is continuous on $X, f\left(x_{\alpha}\right)=f(x)$ for large $\alpha$, and hence $f\left(y_{\alpha}\right)=f(y)$, a contradiction.

As a corollary we have the following refined version of Theorem 6.2.

COROLlaRY. Let $A$ be a ring of characteristic $p$, in which every element satisfies $a^{p^{n}}=a$, and in which every ideal is countably generated. There exists a locally compact zero-dimensional space $X$, with a closed subset $X_{k}$ for each divisor $k$ of $n$, such that $A$ is isomorphic to the set of all continuous functions from $X$ to $G F\left(p^{n}\right)$, vanishing outside a compact set, and on $X_{k}$ taking values in $G F\left(p^{k}\right)$.

A similar refinement of the corollary to Theorem 6.1 is possible, but as a matter of fact here a stronger theorem can be proved. Although the technique of Kronecker products and automorphisms seems to be inapplicable to the noncommutative case, over a real closed field we can give a direct proof of the following result.

THEOREM 8.2. Let $A$ be an algebraic algebra without nilpotent elements over a real closed field $K$, and suppose that every ideal in $A$ is countably generated. There exists a locally compact zero-dimensional space $X$ with closed subsets $Y, Z(X \supset Y \supset Z)$, such that $A$ is isomorphic to the set of all continuous functions vanishing outside a compact set from $X$ to the quaternions $K(i, j, k)$, restricted on $Y$ to values in $K(i)$, and further restricted on $Z$ to values in $K$.

Proof. The proof is based on ideas already used, and we shall merely outline it. The points of $X$ where the residue field is $K$ form a closed set $Z$. We express $X-Z$ as a union of disjoint open compact sets $W_{r}$. For each $r$ we build an element $i_{r}$ vanishing outside $W_{r}$, and satisfying $i_{r}^{2}=-1$ in $W_{r}$ (cf. the proof of Theorem 5.2). Again the points where the residue field is isomorphic to $K$ 
or $K(i)$ form a closed set $Y$ whose complement we express as a union of disjoint open compact sets $V_{s}$, and we may suppose that this decomposition refines the one given by the $W$ 's. In each $V_{\mathrm{s}}$ we supplement the element $i_{r}$ already chosen with quaternion units $j_{s}, k_{s}$. By referring the general element of $A$ to the $i$ 's, $j$ 's, and $k$ 's, we set up a well defined functional representation. There is no difficulty in verifying that the resulting functions are continuous, and that we get all continuous functions satisfying the statement in the theorem.

We shall conclude this section with an example of a ring admitting a representation by Theorem 6.1 , but not one by Theorem 8.1. Let $X=X_{\omega} \times X_{\Omega}$ be the space described at the end of $\S 7, \theta$ the homeomorphism there defined, and $A$ the ring of all continuous functions $f$ from $X$ to $G F(4)$ satisfying

$$
f[\theta(x)]=[f(x)]^{2} .
$$

If $A$ were representable as a set of continuous functions on its own structure space $Y$, then to each point $y \in Y$ we could pick that point $x \in X$ for which $f(x)=f(y)$ for all $f \in A$ (it being readily seen that one of the two eligible points must have this property). The totality of points of $X$ thus selected would constitute a fundamental domain, known not to exist.

We may modify this example by taking $B$ to be the set of all functions satisfying (4) and vanishing at the point $(\omega, \Omega)$. The structure space of $B$ is $X-(\omega, \Omega)$, with all pairs $x$ and $\theta(x)$ identified. Every residue field is $G F(4)$, but $B$ cannot admit $G F(4)$ as a field of operators. For if the latter were the case, $B$ would be representable as a set of continuous functions on its own structure space, and we would be led again to the forbidden fundamental domain. The ring $B$ is the example referred to in the remarks after Theorem 5.2 .

9. Banach algebras. In this section we shall consider commutative Banach algebras; these are normed rings in the sense of Gelfand [6], except that we do not assume a unit element. Moreover we shall only suppose that multiplication by real scalars is defined; and to emphasize this, we shall employ the term "real Banach algebra," although it may very well happen that complex scalar multiplication is introducible. We shall call $A$ a real Banach *algebra if there is defined an operation * which satisfies

$$
(\lambda f+g)^{*}=\lambda f^{*}+g^{*}, \quad(f g)^{*}=g^{*} f^{*}, \quad f^{* *}=f,
$$

and $\left({ }^{12}\right)$

(12) This assumption is essentially the same as one made in a different context in [1]. It is somewhat stronger than Gelfand and Neumark's axiom: $\left\|f f^{*}\right\|=\|f\|\left\|f^{*}\right\|$. Since our assumptions do not preclude the possibility that $f^{*}=f$ for every $f$, this latter axiom would not be adequate to yield a result like Theorem 9.1. A counter-example is furnished by the ring of functions analytic in the unit circle and continuous on the boundary. 


$$
\|f\|^{2} \leqq\left\|f f^{*}+g g^{*}\right\|
$$

where $\lambda$ is a real number and $f, g$ are arbitrary elements of $A$.

If $A$ is a real commutative Banach *-algebra and $M$ a maximal ideal in $A, A-M$ may be either the reals or complexes, and there is nothing in our assumptions to prevent a more or less arbitrary admixture of both. Our purpose is to get a representation theorem allowing for such an admixture. Before doing so we shall give an example to show that complex scalar multiplication need not be introducible even if every residue field is the complex numbers. This is in notable contrast with Theorem 5.2, a contrast explained by the fact that for Banach algebras the structure space need of course not be zerodimensional.

Let $A_{1}$ be the set of all continuous complex-valued functions $f(t)$, defined for $0 \leqq t \leqq 1$, subject to $\left({ }^{13}\right)$

$$
f(0)^{c}=f(1) .
$$

We define addition and multiplication as usual, and set $f^{*}(t)=f(t)^{c}$. Evidently $A_{1}$ is a real Banach *-algebra, and every residue field is the complex numbers. However complex scalars cannot be introduced for if so there would exist (since $A_{1}$ contains a unit) a continuous function $j$ in $A_{1}$ for which $j(t)^{2}$ $=-1$ for all $t$. Such a function could only have the values $\pm i$, and since it is defined on a connected set, it must assume one of these values once for all. This contradicts (6).

We shall now obtain a representation theorem for real Banach *-algebras, combining certain features of Theorem 6.1 and [7]. The words in square parentheses are to be inserted in the event that $A$ does not have a unit.

THEOREM 9.1. Let $A$ be a commutative real Banach *-algebra with [without] $a$ unit. Then there exists a [locally] compact Hausdorff space $X$ having an involutory homeomorphism $\sigma$ such that $A$ is isomorphic to the ring of all those continuous complex-valued functions on $X$ [vanishing at infinity] which satisfy $f[\sigma(x)]=f(x)^{c}$ for $x \in X$. Furthermore if $\|\cdot\|$ is the norm in $A$, then

$$
\|f\|=\sup _{x \in X}|f(x)|
$$

and the *operation is identified as $f^{*}(x)=f(x)^{c}$.

Proof. Let $A^{\prime}$ be the class of all pairs of elements of $A$, with the following rules of operation:

$$
\begin{aligned}
(f, g)+(u, v) & =(f+u, g+v), & (f, g)(u, v) & =(f u-g v, g u+f v), \\
(f, g)^{*} & =\left(f^{*},-g^{*}\right), & v(f, g) & =(\lambda f-\mu g, \lambda g+\mu f),
\end{aligned}
$$

where in the last equation we are defining scalar multiplication by the complex number $\nu=\lambda+i \mu$. We next define a norm in $A^{\prime}$ :

(18) The notation $x^{\varepsilon}$ will denote the complex conjugate of $x$. 


$$
\|f, g\|=\sup _{0 \leqq \theta \leqq 2 \pi}\|f \cos \theta+g \sin \theta\|,
$$

the old norm of $A$ appearing on the right. There is no danger of confusing the two because the new norm bars always enclose a comma. From (8) we obtain

$$
\|f\|,\|g\| \leqq\|f, g\| \leqq\left(\|f\|^{2}+\|g\|^{2}\right)^{1 / 2} .
$$

From (9) and the completeness of $A$ we infer that $A^{\prime}$ is complete. $A$ is embedded in $A^{\prime}$ with preservation of norm, via the correspondence $f \rightarrow(f, 0)$. A simple computation shows that the norm in $A^{\prime}$ is homogeneous, that is,

$$
\|\nu(f, g)\|=|\nu|\|f, g\|
$$

where $\nu$ is a complex number. Again

$$
\begin{aligned}
\|(f, g)(u, v)\| & =\sup \|(f \cos \theta+g \sin \theta) u+(f \sin \theta-g \cos \theta) v\| \\
& \leqq\|f, g\|(\|u\|+\|v\|) \leqq 2\|f, g\|\|u, v\| .
\end{aligned}
$$

From (5) with $g=0$ we obtain $\|f\|=\|f *\|$, hence $\|(f, g) *\|=\|f, g\|$, and hence

$$
\begin{aligned}
2\left\|(f, g)^{*}(f, g)\right\| & =2\left\|f f^{*}+g g^{*}\right\| \geqq\|f\|^{2}+\|g\|^{2} \\
& \geqq\|f, g\|^{2}=\|f, g\|\left\|(f, g)^{*}\right\| .
\end{aligned}
$$

Now on the authority of (10) and (11) we refer to [1, Theorem 1 and Corollary] and make the following statement about $A^{\prime}$ : there exists a [locally] compact Hausdorff space $X$ such that $A^{\prime}$ is isomorphic to the algebra of all continuous complex-valued functions on $X$ [vanishing at infinity]; the *-operation is identified by $F^{*}(x)=F(x)^{c}$, and the topology of $A^{\prime}$ is such that a sequence $F_{n}$ approaches 0 in $A^{\prime}$ if and only if $F_{n}(x)$ converges uniformly to 0 on $X$. We can say more about those functions $f$ of $A^{\prime}$ which were originally in $A$. Indeed since (5) implies $\left\|f^{2}\right\|=\|f\|^{2}$, it can be seen that each of the following statements is equivalent to its successor:

$$
\begin{aligned}
& \sup _{x \in X}|f(x)|<1, \quad \lim _{n} \sup _{x}\left|[f(x)]^{n}\right|=0, \\
& \lim \left\|f^{n}\right\|=0, \quad \lim \|f\|^{n}=0, \quad\|f\|<1 .
\end{aligned}
$$

It follows that (7) holds in $A$.

To construct the homeomorphism $\sigma$ we observe that the mapping $(f, g)$ $\rightarrow\left(f^{*}, g^{*}\right)$ is an involutory automorphism and an isometry of $A^{\prime}$. Hence it is induced by an involutory homeomorphism $\sigma$ of $X$. The functions lying in $A$ are precisely those for which this mapping is the same as the ${ }^{*}$-operation of $A^{\prime}$. Thus $A$ consists of all $f$ with $f[\sigma(x)]=f(x)^{c}$. This completes the proof of Theorem 9.1.

To illustrate Theorem 9.1 we refer again to the ring $A_{1}$ defined at the beginning of this section, and remark that for it the space $X$ is homeomorphic to the circumference of a circle, and $\sigma$ is equivalent to a rotation of the circle 
through an angle $\pi$.

We shall conclude with some remarks on the topological questions connected with Theorem 9.1. First if we suppose that the structure space $X$ of $A^{\prime}$ is known, then the possibilities for $A$ are determined entirely by the involutory homeomorphisms $\sigma$ admitted by $X$. Moreover the structure space $Y$ of $A$ can be obtained from $X$ by identifying all pairs $x, \sigma(x)$. If we wish to represent $A$ as a set of functions on $Y$ rather than $X$, we must find a fundamental domain (in the sense of $\S 7$ ) for $X$ and $\sigma$. We may quote Theorem 7.1 whenever it is applicable, but it should be observed that $X$ is not necessarily zero-dimensional in the present context. However there is another simple case where a fundamental domain exists: if $X$ is a subset of the real line. More generally, if $X$ is any ordered set in its order topology, and $G$ is any finite group of homeomorphisms, we can pick a fundamental domain $X_{G}$ by simply selecting the "smallest" point in each orbit.

Another kind of question arises if we suppose that $Y$ is known and inquire what $X$ and $\sigma$ may be. In some cases a fairly complete answer can be given. We shall suppose that $\sigma$ has no fixed points (this is equivalent to the assumption that every residue field of $A$ is the complex numbers), and that $Y$ is arcwise connected and compact. Then the mapping from $X$ to $Y$ is a local homeomorphism. It follows that any component of $X$ must map on all of $Y$, and thus there are two possibilities: either $X$ splits into two open and closed sets each homeomorphic to $Y$, or $X$ is itself connected (and therefore arcwise connected). If we assume further that $Y$ is simply connected, the latter possibility can be ruled out $\left({ }^{14}\right)$. We may summarize as follows.

THEOREM 9.2. Let $A$ be a commutative real Banach *-algebra whose structure space $Y$ is compact, arcwise connected, and simply connected, and suppose that every residue field of $A$ is the complex numbers. Then $A$ is isomorphic to the ring of all continuous complex functions on $Y$.

Still assuming that $\sigma$ has no fixed points, we may also give a complete result for the case where $Y$ is the circumference of a circle. Here $X$ is either two disjoint circles interchanged by $\sigma$, or a single circle with $\sigma$ a rotation through $\pi$.

If $\sigma$ is allowed to have fixed points (that is, if some residue fields are real), the possibilities become more complicated. But a complete result can be given if $Y$ is the unit interval: $X$ must consist of two unit intervals with the corresponding points of a certain closed set identified. Thus in this case a fundamental domain exists, and we may describe the structure of $A$ as follows.

THEOREM 9.3. Let $A$ be a commutative real Banach *-algebra whose structure space $Y$ is homeomorphic to the unit interval. Then $A$ is isomorphic to the ring of all continuous complex functions on $Y$ which assume only real values on a certain closed subset of $Y$.

(14) Cf. S. Eilenberg, Fund. Math. vol. 24 (1935) p. 42, Theorem III. 


\section{BIBLIOGRAPHY}

1. R. Arens, Representation of Banach *algebras, Duke Math. J.vol. 14 (1947) pp. 269-282.

2. - Linear topological division algebras, Bull. Amer. Math. Soc. vol. 53 (1947) pp. 623-630.

3. E. Artin and G. Whaples, The theory of simple rings, Amer. J. Math. vol. 65 (1943) pp. 87-107.

4. B. Brown and N. H. McCoy, Radicals and subdirect sums, Amer. J. Math. vol. 69 (1947) pp. $46-58$.

5. A. Forsythe and N. H. McCoy, The commutativity of certain rings, Bull. Amer. Math. Soc. vol. 52 (1946) pp. 523-526.

6. I. Gelfand, Normierte ringe, Rec. Math. (Mat. Sbornik) N.S. vol. 9 (1941) pp. 1-23.

7. I. Gelfand and M. Neumark, On the imbedding of normed rings into the ring of operators in Hilbert space, Rec. Math. (Mat. Sbornik) N.S. vol. 12 (1943) pp. 197-213.

8. W. Hurewicz and H. Wallman, Dimension theory, Princeton, 1941.

9. N. Jacobson, Theory of rings, Mathematical Surveys, vol. 2, American Mathematical Society, New York, 1943.

10. - The radical and semi-simplicity for arbitrary rings, Amer. J. Math. vol. 67 (1945) pp. 300-320.

11. - Structure theory of simple rings without finiteness assumptions, Trans. Amer. Math. Soc. vol. 57 (1945) pp. 228-245.

12. - A topology for the set of primitive ideals in an arbitrary ring, Proc. Nat. Acad. Sci. U.S.A. vol. 31 (1945) pp. 333-338.

13. - Structure theory for algebraic algebras of bounded degree, Ann. of Math. vol. 46 (1945) pp. 695-707.

14. R. E. Johnson and F. Kiokemeister, The endomorphisms of the total operator domain of an infinite module, Trans. Amer. Math. Soc. vol. 62 (1947) pp. 404-430.

15. I. Kaplansky, Topological methods in valuation theory, Duke Math. J. vol. 14 (1947) pp. 527-541.

16. —, Topological rings, Amer. J. Math. vol. 69 (1947) pp. 153-183.

17. S. Mazur, Sur les anneaux linéaires, C. R. Acad. Sci. Paris vol. 207 (1938) pp. 10251027.

18. N. H. McCoy and D. Montgomery, A representation of generalized Boolean rings, Duke Math. J. vol. 3 (1937) pp. 455-459.

19. N. H. McCoy, Subrings of direct sums, Amer. J. Math. vol. 60 (1938) pp. 374-382.

20. J. v. Neumann, Regular rings, Proc. Nat. Acad. Sci. U.S.A. vol. 22 (1936) pp. 707713.

21. I. E. Segal, The group algebra of a locally compact group, Trans. Amer. Math. Soc. vol. 61 (1947) pp. 69-105.

22. M. H. Stone, The theory of representations for Boolean algebras, Trans. Amer. Math. Soc. vol. 40 (1936) pp. 37-111.

23. - Applications of the theory of Boolean rings to general topology, Trans. Amer. Math. Soc. vol. 41 (1937) pp. 375-481.

Institute for Advanced Study,

Princeton, N. J.

University OF Chicago,

Chicago, Ill. 